\title{
On Least Squares Estimation in a Simple Linear Regression Model with Periodically Correlated Errors: A Cautionary Note
}

\author{
Abdullah A. Smadi ${ }^{1}$ and Nour H. Abu-Afouna ${ }^{2}$ \\ ${ }^{1}$ Yarmouk University, Irbid, Jordan \\ ${ }^{2}$ Princess Noura University, Riyadh, Saudi Arabia
}

\begin{abstract}
In this research the simple linear regression (SLR) model with autocorrelated errors is considered. Traditionally, correlated errors are assumed to follow the autoregressive model of order one $(\mathrm{AR}(1))$. Beside this model we will also study the SLR model with errors following the periodic autoregressive model of order one $(\operatorname{PAR}(1))$. The later model is useful for modeling periodically autocorrelated errors. In particular, it is expected to be useful when the data are seasonal. We investigate the properties of the least squares estimators of the parameters of the simple regression model when the errors are autocorrelated and for various models. In particular, the relative efficiency of those estimates are obtained and compared for the white noise, $\operatorname{AR}(1)$ and PAR(1) models. Also, the generalized least squares estimates for the SLR with PAR(1) errors are derived. The relative efficiency of the intercept and slope estimates based on both methods is investigated via Monte-Carlo simulation. An application on real data set is also provided. It should be emphasized that once there are sufficient evidences that errors are autocorrelated then the type of this autocorrelation should be uncovered. Then estimates of model's parameters should be obtained accordingly, using some method like the generalized least squares but not the ordinary least squares.
\end{abstract}

Zusammenfassung: In diesem Forschung wird das einfache lineare Regressions (SLR)-Modell mit autokorrelierten Fehlern betrachtet. Traditionell wird für korrelierte Fehler angenommen, dass diese einem autoregressiven Modell der Ordnung Eins folgen (AR(1)). Neben diesem Modell werden wir auch das SLR-Modell mit Fehlern untersuchen, die einem periodischen autoregressiven Modell der Ordnung Eins folgen (PAR(1)). Das letztere Modell ist zur Modellierung periodischer autokorrelierter Fehler nützlich. Insbesondere wird erwartet, dass es nützlich ist, falls die Daten saisonal sind. Wir untersuchen die Eigenschaften der Kleinsten Quadrate Schätzer der Parameter des einfachen Regressionsmodell, wenn die Fehler autokorreliert sind. Insbesondere erhalten wir die relative Effizienz dieser Schätzer und vergleichen sie unter weißem Rauschen, AR(1) und PAR(1)-Modellen. Verallgemeinerte Kleinste Quadrate Schätzer für das SLR mit PAR(1) Fehlern werden auch hergeleitet. Die relative Effizienz der Achsenabschnitts- und des Steigungs-Schätzers unter beiden Methoden wird mittels einer MonteCarlo-Simulation untersucht. Eine Anwendung auf echte Daten wird ebenfalls gemacht. Es wird betont, dass, sobald es genügend Hinweise gibt, dass 
die Fehler autokorreliert sind, der Typ dieser Autokorrelation aufgedeckt werden soll. Dann sollten die Schätzer der Modellparameter entsprechend erhalten werden, mit irgendeiner Methode wie die verallgemeinerte Kleinste Quadrate Schätzung, aber nicht die der gewöhnlichen Kleinsten Quadrate.

Keywords: Simple Linear Regression Model, Autoregression, Periodically Correlated Errors, Relative Efficiency.

\section{Introduction}

Regression analysis is a very important statistical method that investigates the relationship between a response variable $Y$ and a set of other variables named as independent variables or predictors $X_{1}, \ldots, X_{p}$. An important objective of the built model is the prediction of $Y$ for given values of the predictors.

The simple linear regression (SLR) model is the simplest regression model in which we have only one predictor $X$. This model, which is common in practice, is written as

$$
Y_{t}=\beta_{0}+\beta_{1} X_{t}+\epsilon_{t}, \quad t=1, \ldots, n,
$$

where $Y_{t}, X_{t}$ are the values of the response and predictor variables in the th trial, respectively, $\beta_{0}$ and $\beta_{1}$ are unknown parameters and $\epsilon_{t}$ are usually assumed to be iid from $\mathrm{N}\left(0, \sigma_{\epsilon}^{2}\right)$ specially for inference purposes. The terms $\left\{\epsilon_{t}\right\}$ satisfying such conditions are named in the time series context as the white noise (WN) process (Wei, 2006). The variable $X$ is usually assumed fixed and non-random. For several predictors, the SLR model generalizes to what is known as the multiple linear regression model. For the estimation of the SLR model there are two common methods, the first is the ordinary least squares (OLS) method, which relies on minimizing the sum of square of errors $\sum \epsilon_{t}^{2}$. For the SLR model (1) the OLS estimators of $\beta_{0}$ and $\beta_{1}$ are

$$
\hat{\beta}_{0}=\bar{Y}-\hat{\beta}_{1} \bar{X}
$$

and

$$
\hat{\beta}_{1}=\frac{\sum_{t}\left(X_{t}-\bar{X}\right)\left(Y_{t}-\bar{Y}\right)}{\sum_{t}\left(X_{t}-\bar{X}\right)^{2}}=\frac{S_{X Y}}{S_{X X}} .
$$

It is known that these estimators are unbiased and best linear unbiased estimators (BLUE). The second method is the maximum likelihood method, which under the assumptions of independence and normality of $\epsilon_{t}$ produces again $\hat{\beta}_{0}$ and $\hat{\beta}_{1}$ above (Kutner, Nachtsheim, Neter, and Li, 2005, p. 31-32).

In turn, the fitted SLR model is written as

$$
\hat{Y}_{t}=\hat{\beta}_{0}+\hat{\beta}_{1} X_{t}
$$

so that the estimated errors or residuals, denoted by $e_{t}$, are defined as $e_{t}=Y_{t}-\hat{Y}_{t}$, $t=1, \ldots, n$. Once, the regression model is fitted, an important step in model building and diagnosis is to check for the assumptions of the model, namely; independence, normality and constant variance of errors. The residuals of the fitted model play a primary role 
for this purpose. Several graphs and tests for residuals may be used to examine those assumptions. These techniques are usually known as residual analysis. In particular, to examine the independence of error terms we use the plot of residuals against time or order (usually named as the residual plot). Besides, an important test specifically designed for testing the lack of randomness in residuals is the Durbin-Watson test. For a detailed account on various methods for the assessment of the assumptions of regression models, see Kutner et al. (2005).

When the assumptions of the regression model are not valid there exist several remedial measures. In particular, when the error terms are correlated, in which case model (1) is named as generalized linear regression (GLR) model, a direct remedial measure is to work with a model that calls for correlated error terms (Kutner et al., 2005, p. 127). A common model of errors in this case is the stationary zero-mean $\operatorname{AR}(1)$ model so that $\epsilon_{t}$ in (1) now satisfies

$$
\epsilon_{t}=\phi \epsilon_{t-1}+u_{t},
$$

where $u_{t}$ are assumed iid $\mathrm{N}\left(0, \sigma_{u}^{2}\right)$ and $|\phi|<1$. When $\phi=0$, this model reduces to the ordinary SLR model. Other possible models for errors can be selected from the wider class of mixed autoregressive moving average (ARMA) models (Box, Jenkins, and Reinsel, 1994).

In the literature, regression models with autocorrelated errors following some ARMA model have been widely studied (Mohammed and Ibazizen, 2008; Huitema and McKean, 2007; Lee and Lund, 2004; Jeske, Bütefisch, and Song, 1996; Zinde-Walsh and Galbraith, 1991). More general results for regression models with correlated errors are considered, for example, by Grenander (1954), Choudhury, Hubata, and Louis (1999) and Yue and Koreisha (2004). In particular, Ohtani (1990) examined the small-sample properties of the generalized least squares (GLS) estimators and tests of individual regression coefficients with autocorrelated errors.

Lee and Lund (2004) considered the OLS and GLS estimation for the linear trend model

$$
Y_{t}=\beta_{1}+\beta_{1} t+\epsilon_{t} .
$$

They have derived explicit formulas for the variances of the OLS and the GLS estimates of $\beta_{0}$ and $\beta_{1}$ with errors following some selected ARMA models.

The GLS estimate of $\beta=\left(\beta_{0}, \beta_{1}\right)^{\prime}$ under the SLR model (1) is

$$
\hat{\beta}_{G L S}=\left(X_{n}^{\prime} \Gamma_{n}^{-1} X_{n}\right)^{-1} X_{n}^{\prime} \Gamma_{n}^{-1} Y_{n},
$$

where $Y_{n}=\left(Y_{1}, \ldots, Y_{n}\right)^{\prime}, X_{n}=\left(1_{n}, X_{i}\right)^{\prime}$ is the design matrix where $1_{n}$ is a $n$-dimensional vector of ones and $\Gamma_{n}$ is the auto-covariance matrix of the errors $\epsilon=\left(\epsilon_{1}, \ldots, \epsilon_{n}\right)^{\prime}$ written as

$$
\Gamma_{n}=\left(\begin{array}{cccc}
\gamma_{0} & \gamma_{1} & \ldots & \gamma_{n-1} \\
\gamma_{1} & \gamma_{0} & \ldots & \gamma_{n-2} \\
\vdots & \vdots & \ddots & \vdots \\
\gamma_{n-1} & \gamma_{n-2} & \ldots & \gamma_{0}
\end{array}\right)
$$

where $\gamma_{k}=\operatorname{cov}\left(\epsilon_{t}, \epsilon_{t-k}\right), k=0,1, \ldots$ Besides, the variance of $\hat{\beta}_{G L S}$ is given by

$$
\operatorname{var}\left(\hat{\beta}_{G L S}\right)=\left(X_{n}^{\prime} \Gamma_{n}^{-1} X_{n}\right)^{-1} .
$$


Grenander (1954) showed that the OLS and GLS estimates are asymptotically equally efficient for SLR with stationary correlated errors. However, once the errors are correlated, the OLS estimates loses its minimum variance property. In a later part of this work, we will see that this fact of deficiency of OLS estimates carries over to the case of periodically correlated errors.

In this article we study the properties of OLS estimates for the parameters of SLR when the errors are periodically correlated. In the time series framework it is found that many real time series exhibit periodic autocorrelations that can not be modelled by ordinary seasonal ARMA models (Tiao and Grupe, 1980; Franses and Paap, 2004; McLeod, 1995). This means that the autocorrelations among successive errors changes from one season to another. For example, assuming that $\left\{Y_{12 k+\nu}\right\}$ is a monthly time series then periodic autocorrelation means that $\operatorname{corr}\left(Y_{12 k+\nu}, Y_{12 k+\nu-1}\right)$ is not constant for $\nu=1, \ldots, 12$. McLeod (1995) suggests a simple graphical method to detect periodic autocorrelations in time series. He also proposed a test of periodically autocorrelated errors. McLeod proposed to apply this test on the residuals resulted from fitting seasonal ARIMA models for some seasonal time series. If significant, this test will indicate that the errors are periodically correlated.

\section{GLR Models with Periodically Correlated Errors}

Assume that $\left\{Y_{t}\right\}$ (and possibly $\left\{X_{t}\right\}$ ) is a seasonal time series with period $\omega$. Then, if the errors in (1) are correlated then they may inhibit some seasonality. In this case there are several approaches to handle this issue. The first is using seasonal ARMA models (see Box et al., 1994) or one can add some extra regressors to (1) to extract the seasonality as for instance seasonal dummy variables or trigonometric functions. The later approach is useful when the seasonality in $\left\{Y_{t}\right\}$ is deterministic and the former is useful when seasonality is stochastic but homogeneous.

An alternative model that is suitable for modelling seasonality is the periodic ARMA (PARMA) models. Writing the time $t$ in terms of the period $\omega$ as $k \omega+\nu$ where $\nu=$ $1, \ldots, \omega$ denotes the season and $k$ denotes the year, the zero-mean $\operatorname{PARMA}_{\omega}(p(\nu), q(\nu))$ model is written as:

$$
\left(1-\phi_{1}(\nu) B-\cdots-\phi_{p(\nu)}(\nu) B^{p(\nu)}\right) \epsilon_{k \omega+\nu}=\left(1-\theta_{1}(\nu) B-\cdots-\theta_{q(\nu)}(\nu) B^{q(\nu)}\right) u_{k \omega+\nu}
$$

where $\left\{u_{k \omega+\nu}\right\}$ is a zero-mean $\mathrm{WN}$ process with periodic variances $\sigma_{u}^{2}(\nu), p(\nu)$ is the $\mathrm{AR}$ order for season $\nu$ and $q(\nu)$ is the MA order for season $\nu$ and $\phi_{1}(\nu), \ldots, \phi_{p(\nu)}(\nu)$ and $\theta_{1}(\nu), \ldots, \theta_{q(\nu)}(\nu)$ are the $\mathrm{AR}$ and MA parameters of season $\nu$, respectively (Franses and Paap, 2004). The periodic autoregressive model (PAR) is a special case of the PARMA model. In (7) setting $q(\nu)=0$ for each $\nu=1, \ldots, \omega$ we get the equation of the $\operatorname{PAR}_{\omega}(p(\nu))$ model. For instance, the zero-mean $\operatorname{PAR}_{\omega}(1)$ model can be written as

$$
\epsilon_{k \omega+\nu}=\phi_{1}(\nu) \epsilon_{k \omega+\nu-1}+u_{k \omega+\nu}, \quad \nu=1, \ldots, \omega
$$

In fact, this equation can be written as $\omega$ equations. For instance, the zero-mean 
$\mathrm{PAR}_{4}(1)$ model can be written as

$$
\begin{aligned}
& \epsilon_{4 k+1}=\phi_{1}(1) \epsilon_{4(k-1)+4}+u_{4 k+1} \\
& \epsilon_{4 k+2}=\phi_{1}(2) \epsilon_{4 k+1}+u_{4 k+2} \\
& \epsilon_{4 k+3}=\phi_{1}(3) \epsilon_{4 k+2}+u_{4 k+3} \\
& \epsilon_{4 k+4}=\phi_{1}(4) \epsilon_{4 k+3}+u_{4 k+4} .
\end{aligned}
$$

PARMA models are not stationary in the ordinary weak sense. They are rather examined for a weaker type of stationarity named as periodic stationarity. This means that the mean and the variance of the time series is constant for each season and periodic with period $\omega$ and the autocovariance function depends on the time lag and season only (Ula and Smadi, 1997). For example, the $\mathrm{PAR}_{4}(1)$ model above is periodic stationary if $\left|\prod_{\nu=1}^{4} \phi_{1}(\nu)\right|<$ 1 (Obeysekera and Salas, 1986). The essence of PARMA models is that it is suitable for modelling periodic correlations. The first motivations for those models started in hydrology then found any applications in economics and other areas (Obeysekera and Salas, 1986; Franses and Paap, 2004).

If the period $\omega=1$, then the $\operatorname{PARMA}_{\omega}(p(\nu), q(\nu))$ and $\operatorname{PAR}_{\omega}(1)$ models reduce, respectively to the ordinary $\operatorname{ARMA}(p, q)$ and $\operatorname{AR}(1)$ models. For more details on PARMA models see for example Franses and Paap (2004).

Therefore, the main idea in this research is to propose the idea of periodically correlated errors in SLR model through using the PAR(1) model, that is

$$
Y_{k \omega+\nu}=\beta_{0}+\beta_{1} X_{k \omega+\nu}+\epsilon_{k \omega+\nu}
$$

with errors following (8). In the next section the properties of the OLS estimates of $\beta_{0}$ and $\beta_{1}$ are to be investigated. Later on, the GLS estimates of $\beta_{o}$ and $\beta_{1}$ are also derived.

\section{Properties of OLS Estimates with Correlated Errors}

The method of moments is one of the most common methods of estimation in statistical inference. It is also common in the context of time series analysis. Assuming that $\left\{U_{k \omega+\nu}\right\}$ is a periodic stationary process, then the seasonal autocorrelation function depends on the time lag and season only and is defined as

$$
\rho_{j}(\nu)=\operatorname{corr}\left(U_{k \omega+\nu}, U_{k \omega+\nu-j}\right)=\frac{\gamma_{j}(\nu)}{\sqrt{\gamma_{0}(\nu) \gamma_{0}(\nu-j)}}, \quad j=0,1, \ldots,
$$

where $\gamma_{j}(\nu)=\operatorname{cov}\left(U_{k \omega+\nu}, U_{k \omega+\nu-j}\right)$ denotes the seasonal autocovariance function (SACVF) and $\gamma_{0}(\nu)$ denotes the variance of the process for season $\nu$ and time lag $j$.

Based on an observed realization $u_{1}, \ldots, u_{m \omega}$ the moment estimator of $\rho_{j}(\nu)$ is

$$
r_{j}(\nu)=\frac{C_{j}(\nu)}{\sqrt{C_{0}(\nu) C_{0}(\nu-j)}}
$$

where

$$
C_{0}(\nu)=\frac{1}{m-1} \sum_{i=0}^{m-1}\left(u_{i \omega+\nu}-\bar{u}_{\nu}\right)^{2}
$$


and

$$
C_{j}(\nu)=\frac{1}{m-1} \sum_{i=0}^{m-1}\left(u_{i \omega+\nu}-\bar{u}_{\nu}\right)\left(u_{i \omega+\nu-j}-\bar{u}_{\nu-j}\right),
$$

where $\bar{u}_{\nu}$ is the sample mean of data in season $\nu$ and $\mathrm{m}$ is the number of years of data. It can be shown that $r_{j}(\nu)$ are asymptotically unbiased and consistent estimators of $\rho_{j}(\nu)$ (McLeod, 1995).

As far as the $\mathrm{PAR}_{\omega}(1)$ is considered, it can be proved that the first lag autocorrelations are given by:

$$
\rho_{1}(\nu)=\phi_{1}(\nu) \sqrt{\frac{\gamma_{0}(\nu-1)}{\gamma_{0}(\nu)}},
$$

for $\nu=1, \ldots, \omega$. Note that in this case the first order autocorrelation are not the same as AR parameters but a function of them unless all $\gamma_{0}(\nu)$ are equal. For a given $\operatorname{PAR}_{\omega}(1)$ model with known $\phi_{1}(\nu)$ and $\sigma_{u}^{2}(\nu), \nu=1, \ldots, \omega$, then $\gamma_{0}(\nu)$ can be obtained, for all $\nu$, by solving the system of equations

$$
\gamma_{0}(\nu)=\left(\phi_{1}(\nu)\right)^{2} \gamma_{0}(\nu-1)+\sigma_{u}^{2}(\nu), \quad \nu=1, \ldots, \omega
$$

Then $\rho_{1}(\nu)$ can be computed using (5).

For the computation of $\gamma_{j}(\nu)$ for $\operatorname{PAR}_{\omega}(1)$ models the following theorem is useful.

Theorem 1: If $\left\{\epsilon_{k \omega+\nu}\right\}$ follows a periodic stationary $\operatorname{PAR}_{\omega}(1)$ model (8), then

$$
\begin{aligned}
\gamma_{j}(\nu) & =\phi_{1}(\nu) \gamma_{j-1}(\nu-1) \\
& =\phi_{1}(\nu) \phi_{1}(\nu-1) \phi_{1}(\nu-2) \cdots \phi_{1}(\nu-j+1) \gamma_{0}(\nu-j) .
\end{aligned}
$$

Proof: It is easy to show that for $j=1$,

$$
\begin{aligned}
\gamma_{1}(\nu) & =\operatorname{cov}\left(\epsilon_{k \omega+\nu}, \epsilon_{k \omega+\nu-1}\right) \\
& =\operatorname{cov}\left(\phi_{1}(\nu) \epsilon_{k \omega+\nu-1}+u_{k \omega+\nu}, \epsilon_{k \omega+\nu-1}\right) \\
& =\phi_{1}(\nu) \gamma_{0}(\nu-1) .
\end{aligned}
$$

Thus for $\mathrm{j}=2$,

$$
\begin{aligned}
\gamma_{2}(\nu) & =\operatorname{cov}\left(\epsilon_{k \omega+\nu}, \epsilon_{k \omega+\nu-2}\right) \\
& =\operatorname{cov}\left(\phi_{1}(\nu) \epsilon_{k \omega+\nu-1}+u_{k \omega+\nu}, \epsilon_{k \omega+\nu-2}\right) \\
& =\phi_{1}(\nu) \gamma_{1}(\nu-1) \\
& =\phi_{1}(\nu) \phi_{1}(\nu-1) \gamma_{0}(\nu-2) .
\end{aligned}
$$

Iterating for $k$ we get (12).

When $\epsilon_{t}$ is WN we have seen that $\hat{\beta}_{0}$ and $\hat{\beta}_{1}$ are the BLUE and $\operatorname{var}\left(\hat{\beta}_{0}\right)$ and $\operatorname{var}\left(\hat{\beta}_{1}\right)$ are (Kutner et al., 2005)

$$
\operatorname{var}\left(\hat{\beta}_{0}\right)=\frac{\sigma_{\epsilon}^{2}}{S_{X X}}\left[\frac{S_{X X}}{n}+\bar{X}^{2}\right]
$$

and

$$
\operatorname{var}\left(\hat{\beta}_{1}\right)=\frac{\sigma_{\epsilon}^{2}}{S_{X X}}
$$


Now, the following theorem is useful for the computation of $\operatorname{var}\left(\hat{\beta}_{0}\right)$ and $\operatorname{var}\left(\hat{\beta}_{1}\right)$ for errors following any stationary stochastic process and in particular the $\mathrm{AR}(1)$ model.

Theorem 2: For the GLR model (1) with $\left\{\epsilon_{t}\right\}$ being any stationary stochastic process with mean zero and ACVF $\gamma_{k}$, then $\hat{\beta}_{0}$ and $\hat{\beta}_{1}$ are unbiased and

$$
\begin{aligned}
& \operatorname{var}\left(\hat{\beta}_{0}\right)=\sum_{t=1}^{n} c_{t}^{2} \gamma_{0}+2 \sum_{t>j}^{n} c_{t} c_{j} \gamma_{t-j} \\
& \operatorname{var}\left(\hat{\beta}_{1}\right)=\sum_{t=1}^{n} b_{t}^{2} \gamma_{0}+2 \sum_{t>j}^{n} b_{t} b_{j} \gamma_{t-j}
\end{aligned}
$$

with

$$
c_{t}=\frac{1}{n-b_{t} \bar{X}} \quad \text { and } \quad b_{t}=\frac{X_{t}-\bar{X}}{S_{X X}} .
$$

Proof: It can easily be shown that

$$
\hat{\beta}_{0}=\sum_{t=1}^{n} c_{t} Y_{t} \quad \text { and } \quad \hat{\beta}_{1}=\sum_{t=1}^{n} b_{t} Y_{t},
$$

where $c_{t}$ and $b_{t}$ are given by (17). Thus,

$$
\mathrm{E}\left(\hat{\beta}_{0}\right)=\sum_{t=1}^{n} c_{t} \mathrm{E}\left(Y_{t}\right)=\sum_{t=1}^{n} c_{t}\left(\beta_{0}+\beta_{1} X_{t}\right)=\beta_{0} \sum_{t=1}^{n} c_{t}+\beta_{1} \sum_{t=1}^{n} c_{t} X_{t} .
$$

Because, $\sum_{t=1}^{n} c_{t}=1$ and $\sum_{t=1}^{n} c_{t} X_{t}=0$ then $\mathrm{E}\left(\hat{\beta}_{0}\right)=\beta_{0}$. Similarly we can show that $\mathrm{E}\left(\hat{\beta}_{1}\right)=\beta_{1}$. The results in (15) and (16) are direct from the fact that $\hat{\beta}_{0}$ and $\hat{\beta}_{1}$ are linear functions in $Y_{1}, \ldots, Y_{n}$ with coefficients as in (17).

Corollary 1: For the GLR model (1) with $\left\{\epsilon_{t}\right\}$ following the zero-mean AR(1) model (2) with $|\phi|<1$ then $\hat{\beta}_{0}$ and $\hat{\beta}_{1}$ are unbiased and

$$
\begin{gathered}
\operatorname{var}\left(\hat{\beta}_{0}\right)=\frac{\sigma_{u}^{2}}{(1-\phi 2) S_{X X}}\left[\frac{S_{X X}}{n}+\bar{X}^{2}+2 \sum_{t>j}^{n} K_{t j} \phi^{t-j}\right] \\
\operatorname{var}\left(\hat{\beta}_{1}\right)=\frac{\sigma_{u}^{2}}{\left.1-\phi^{2}\right) S_{X X}}\left[1+\frac{2}{S_{X X}} \sum_{t>j}^{n} M_{t j} \phi^{t-j}\right],
\end{gathered}
$$

where

$$
M_{t j}=\left(X_{t}-\bar{X}\right)\left(X_{j}-\bar{X}\right)
$$

and

$$
K_{t j}=\frac{S_{X X}}{n^{2}}-\frac{\bar{X}}{n}\left(\left(X_{t}-\bar{X}\right)+\left(X_{j}-\bar{X}\right)\right)+\frac{\bar{X}^{2}}{S_{X X}}\left(X_{t}-\bar{X}\right)\left(X_{j}-\bar{X}\right) .
$$

It can be seen that when $\phi=0$, then (18) and (19) reduce to the formulas of $\operatorname{var}\left(\hat{\beta}_{0}\right)$ and $\operatorname{var}\left(\hat{\beta}_{1}\right)$ for WN errors given by (13) and (14). For $\phi \neq 0$ the two sets of formulas 
will be compared later on. Now, the following theorem generalizes Theorem 2 to GLR models as (1) with periodically correlated errors.

Theorem 3: For the GLR model (1) with errors following a zero-mean $\mathrm{PAR}_{\omega}(1)$ model (8) with $\left|\prod_{\nu=1}^{4} \phi_{1}(\nu)\right|<1$, then $\hat{\beta}_{0}$ and $\hat{\beta}_{1}$ are unbiased and

$$
\begin{aligned}
\operatorname{var}\left(\hat{\beta}_{0}\right)= & \sum_{\nu=1}^{\omega} \gamma_{0}(\nu) \sum_{k=1}^{m} c_{(k-1) \omega+\nu}^{2} \\
& +2 \sum_{\nu=1}^{\omega} \sum_{k=1}^{m} \sum_{j=1}^{(k-1) \omega+\nu-1} c_{(k-1) \omega+\nu} c_{j} \gamma_{(k-1) \omega+\nu-j}(\nu) \mathrm{I}_{((k-1) \omega+\nu-j \geq 1)} \\
\operatorname{var}\left(\hat{\beta}_{1}\right)= & \sum_{\nu=1}^{\omega} \gamma_{0}(\nu) \sum_{k=1}^{m} b_{(k-1) \omega+\nu}^{2} \\
& +2 \sum_{\nu=1}^{\omega} \sum_{k=1}^{m} \sum_{j=1}^{(k-1) \omega+\nu-1} b_{(k-1) \omega+\nu} b_{j} \gamma_{(k-1) \omega+\nu-j}(\nu) \mathrm{I}_{((k-1) \omega+\nu-j \geq 1)},
\end{aligned}
$$

where

$$
c_{(k-1) \omega+\nu}=\frac{1}{n}-b_{(k-1) \omega+\nu} \bar{X} \quad \text { and } \quad b_{(k-1) \omega+\nu}=\frac{X_{(k-1) \omega+\nu}-\bar{X}}{S_{X X}} .
$$

Proof: The unbiasedness of $\hat{\beta}_{0}$ and $\hat{\beta}_{1}$ can be proved in a similar manner as Theorem 2 . Now we want to prove (22) and (23). We know that

$$
\operatorname{var}\left(\hat{\beta}_{0}\right)=\sum_{t=1}^{n} c_{t}^{2} \operatorname{var}\left(\epsilon_{t}\right)+2 \sum_{t>j}^{n} c_{t} c_{j} \operatorname{cov}\left(\epsilon_{t}, \epsilon_{j}\right),
$$

where $c_{t}$ is defined in (17). Now, assuming $n=m \omega$ where $m$ is the number of years of data, and writing $t$ as $(k-1) \omega+\nu$, then $(25)$ reduces to

$$
\begin{aligned}
\operatorname{var}\left(\hat{\beta}_{0}\right)= & \sum_{\nu=1}^{\omega} \gamma_{0}(\nu) \sum_{k=1}^{m} c_{(k-1) \omega+\nu}^{2} \\
& +2 \sum_{\nu=1}^{\omega} \sum_{k=1}^{m} \sum_{j=1}^{(k-1) \omega+\nu-1} c_{(k-1) \omega+\nu} c_{j} \gamma_{(k-1) \omega+\nu-j}(\nu) \mathrm{I}_{((k-1) \omega+\nu-j \geq 1)},
\end{aligned}
$$

where $\mathrm{I}_{A}$ is the indicator function for the set $A$. The results for $\operatorname{var}\left(\hat{\beta}_{1}\right)$ in (23) can similarly be derived.

In fact, Theorem 3 is valid for all periodic-stationary processes with seasonal ACVF $\gamma_{k}(\nu)$.

\section{The Relative Efficiency for Correlated Errors}

It is known that if $\hat{\theta}_{1}$ and $\hat{\theta}_{2}$ are two unbiased estimators of $\theta$, then the relative efficiency of $\hat{\theta}_{1}$ with respect to $\hat{\theta}_{2}$ is (Rohatgi, 1984, p. 193)

$$
\operatorname{RE}\left(\hat{\theta}_{1}, \hat{\theta}_{2}\right)=\frac{\operatorname{MSE}\left(\hat{\theta}_{2}\right)}{\operatorname{MSE}\left(\hat{\theta}_{1}\right)}=\frac{\operatorname{var}\left(\hat{\theta}_{2}\right)}{\operatorname{var}\left(\hat{\theta}_{1}\right)} .
$$


Therefore, for the SLR model as $\hat{\beta}_{0}$ and $\hat{\beta}_{1}$ are unbiased estimators for $\beta_{0}$ and $\beta_{1}$ for errors following WN, $\operatorname{AR}(1)$ and $\operatorname{PAR}(1)$ models, then we can compare the efficiency of these estimators using (26). For simplicity, the comparison will be carried out with the WN model being the reference case. Those relative efficiencies are easily obtained from the results in the previous section and are explicitly given below.

Corollary 2: The relative efficiency of $\hat{\beta}_{0}$ and $\hat{\beta}_{1}$ for errors following the $\operatorname{AR}(1)$ model with respect to the standard SLR model (i.e. WN errors) are

$$
\begin{aligned}
& \operatorname{RE}\left[\hat{\beta}_{0}(\operatorname{AR}(1)), \hat{\beta}_{0}(\mathrm{WN})\right]=\frac{\left(1-\phi^{2}\right)\left(\frac{S_{X X}}{n}+\bar{X}^{2}\right)}{\frac{S_{X X}}{n}+\bar{X}^{2}+2 \sum_{t>j}^{n} K_{t j} \phi^{t-j}} \\
& \operatorname{RE}\left[\hat{\beta}_{1}(\operatorname{AR}(1)), \hat{\beta}_{1}(\mathrm{WN})\right]=\frac{1-\phi^{2}}{1+2 S_{X X} \sum_{t>j}^{n} M_{t j} \phi^{t-j}}
\end{aligned}
$$

with $K_{t j}$ and $M_{t j}$ given in (20) and (21), respectively.

Corollary 3: The relative efficiency of $\hat{\beta}_{0}$ and $\hat{\beta}_{1}$ for errors following the $\mathrm{PAR}_{\omega}(1)$ model with respect to the standard SLR model are

$$
\begin{aligned}
& \operatorname{RE}\left[\hat{\beta}_{0}(\operatorname{PAR}(1)), \hat{\beta}_{0}(\mathrm{WN})\right] \\
& =\frac{\frac{\sigma^{2}}{S_{X X}}\left(\frac{S_{X X}}{n}+\bar{X}^{2}\right)}{\sum_{\nu=1}^{\omega} \gamma_{0}(\nu) \sum_{k=1}^{m} c_{(k-1) \omega+\nu}^{2}+2 \sum_{\nu=1}^{\omega} \sum_{k=1}^{m} \sum_{j=1}^{(k-1) \omega+\nu-1} c_{(k-1) \omega+\nu} c_{j} \gamma_{(k-1) \omega+\nu-j}(\nu) \mathrm{I}_{((k-1) \omega+\nu-j \geq 1)}}
\end{aligned}
$$

$$
\begin{aligned}
& \operatorname{RE}\left[\hat{\beta}_{1}(\operatorname{PAR}(1)), \hat{\beta}_{1}(\mathrm{WN})\right] \\
= & \frac{\frac{\sigma^{2}}{S_{X X}}}{\sum_{\nu=1}^{\omega} \gamma_{0}(\nu) \sum_{k=1}^{m} b_{(k-1) \omega+\nu}^{2}+2 \sum_{\nu=1}^{\omega} \sum_{k=1}^{m} \sum_{j=1}^{(k-1) \omega+\nu-1} b_{(k-1) \omega+\nu} b_{j} \gamma_{(k-1) \omega+\nu-j}(\nu) \mathrm{I}_{((k-1) \omega+\nu-j \geq 1)}}
\end{aligned}
$$

with $c_{(k-1) \omega+\nu}$ and $b_{(k-1) \omega+\nu}$ given in (24).

Now, we illustrate Corollaries 2 and 3 through the following example.

Example 1: Consider the linear trend model (3) with $n=100$ and $\epsilon_{t}$ from

(a) WN model with $\sigma_{\epsilon}^{2}=16$, 
(b) $\operatorname{AR}(1)$ model with $\phi=-0.8,-0.6,-0.4,-0.2,0,0.2,0.4,0.6,0.8$ and $\gamma_{0}=\sigma_{\epsilon}^{2}=$ 16 ,

(c) $\mathrm{PAR}_{4}(1)$ with $\prod_{\nu=1}^{4} \phi_{1}(\nu)=-0.8,-0.6,-0.4,-0.2,0,0.2,0.4,0.6,0.8$ and $\phi_{1}(\nu)$ values as given in the first column of Table 2 below and $\gamma_{0}(\nu)=16, \nu=1, \ldots, 4$.

Using an R-code written by the authors, the relative efficiencies are computed and summarized in Tables 1 to 3 . To make the comparison more accurate we have fixed $\operatorname{var}\left(\epsilon_{t}\right)$ for all error models. Besides, we have selected the $\phi$ 's for the AR(1) model in (b) satisfying the stationarity condition $|\phi|<1$ and for the $\mathrm{PAR}_{4}(1)$ models in (c) satisfying the periodic stationarity condition $\left|\prod \phi_{1}(\nu)\right|<1$. Also, we have selected $\prod \phi_{1}(\nu)$ in (c) as the same values of $\phi$ for the AR(1) models in (b). It is worth mentioning that in general in $\operatorname{PAR}(1)$ models, $\phi_{1}(\nu)$ can take any real value subject to the condition that $\left|\prod \phi_{1}(\nu)\right|<1$. However, in this example as $\gamma_{0}(\nu)$ is fixed for all seasons, then in view of (11), $\phi_{1}(\nu)$ equals $\rho_{1}(\nu)$. This explains the choice of the $\phi_{1}(\nu)$ values in this example from $(-1,1)$.

In view of Table 1 we can see that in terms of efficiency, the LS estimators $\hat{\beta}_{0}$ and $\hat{\beta}_{1}$ based on the AR(1) model (b) are more (less) efficient than based on WN model when $\phi<$ $0(\phi>0)$. We in fact conclude from this result that when the errors are autocorrelated via an $\operatorname{AR}(1)$ scheme, then when the autocorrelation is positive (negative) then the standard formulas for $\operatorname{var}\left(\hat{\beta}_{0}\right)$ and $\operatorname{var}\left(\hat{\beta}_{1}\right)$ given by (13) and (14), respectively, do underestimate (overestimate) their true values. Besides, as the autocorrelation becomes stronger the gaps between the estimated and true variances of $\hat{\beta}_{0}$ and $\hat{\beta}_{1}$ increase. Therefore, if the errors are actually autocorrelated but ignoring this fact and using the standard formulas for $\operatorname{var}\left(\hat{\beta}_{0}\right)$ and $\operatorname{var}\left(\hat{\beta}_{1}\right)$ then all subsequent results, including prediction, are inaccurate (see, for example, Lee and Lund, 2004).

\begin{tabular}{|c|c|c|}
\hline$\phi$ & $\hat{\beta}_{0}$ & $\hat{\beta}_{1}$ \\
\hline-0.8 & 8.004 & 7.810 \\
\hline-0.6 & 3.812 & 3.775 \\
\hline-0.4 & 2.278 & 2.267 \\
\hline-0.2 & 1.484 & 1.481 \\
\hline 0.0 & 1.000 & 1.000 \\
\hline 0.2 & 0.674 & 0.675 \\
\hline 0.4 & 0.439 & 0.440 \\
\hline 0.6 & 0.262 & 0.264 \\
\hline 0.8 & 0.123 & 0.126 \\
\hline
\end{tabular}

When the errors follow the $\mathrm{PAR}_{4}(1)$ model (c), that is the errors are periodically autocorrelated, then $\operatorname{var}\left(\hat{\beta}_{0}\right)$ and $\operatorname{var}\left(\hat{\beta}_{1}\right)$ do also differ from their standard formulas in (13) and (14). Specifically, it can be seen in Table 2 and 3 that the relative efficiency of $\hat{\beta}_{0}$ and $\hat{\beta}_{1}$ between the WN errors and the $\mathrm{PAR}_{4}(1)$ model differ from one. However, there is no specific pattern in the relative efficiencies in view of $\prod \phi_{1}(\nu)$ as seen in Table 2.

Finally it is worth mentioning that the relative efficiencies for $\hat{\beta}_{0}$ and $\hat{\beta}_{1}$ were very close to each others and have the same direction in all selected cases. 
Table 2: Relative efficiencies of $\hat{\beta}_{0}$ and $\hat{\beta}_{1}$ for $\mathrm{WN} / \mathrm{PAR}_{4}(1)$.

\begin{tabular}{rrrrrrr}
\hline \multicolumn{2}{c}{$\phi_{1}(\nu)$} & & $\prod \phi_{1}(\nu)$ & $\hat{\beta}_{0}$ & $\hat{\beta}_{1}$ \\
\hline-0.99 & 0.95 & 0.9 & 0.9451 & -0.8 & 3.673 & 3.216 \\
0.9 & -0.8 & 0.9 & 0.9259 & -0.6 & 1.730 & 1.693 \\
0.7 & 0.9 & -0.8 & 0.7937 & -0.4 & 1.758 & 1.764 \\
-0.6 & 0.7 & -0.9 & -0.5291 & -0.2 & 3.142 & 3.118 \\
0 & 0 & 0 & 0 & 0.0 & 1.000 & 1.000 \\
0.6 & -0.7 & -0.9 & 0.5291 & 0.2 & 0.790 & 0.789 \\
-0.7 & -0.9 & -0.8 & -0.7937 & 0.4 & 8.942 & 8.748 \\
0.9 & 0.8 & 0.9 & 0.9259 & 0.6 & 0.079 & 0.083 \\
-0.99 & 0.95 & 0.9 & -0.9451 & 0.8 & 0.173 & 0.209 \\
\hline
\end{tabular}

Table 3: Relative efficiencies of $\hat{\beta}_{0}$ and $\hat{\beta}_{1}$ for $\operatorname{AR}(1) / \operatorname{PAR}_{4}(1)$.

\begin{tabular}{rrrrrrr}
\hline \multicolumn{2}{c}{$\phi_{1}(\nu)$} & & $\prod \phi_{1}(\nu)$ & $\hat{\beta}_{0}$ & $\hat{\beta}_{1}$ \\
\hline-0.9 & 1.5 & 0.7 & 0.8466 & -0.8 & 0.459 & 0.412 \\
0.9 & 0.8 & 1.7 & -0.4902 & -0.6 & 0.454 & 0.448 \\
1.4 & -1.1 & 0.4 & 0.6494 & -0.4 & 0.772 & 0.778 \\
0.6 & 0.7 & -0.4 & 1.1905 & -0.2 & 2.117 & 2.105 \\
0 & 0 & 0 & 0 & 0.0 & 1.000 & 1.000 \\
1.9 & 0.8 & 0.7 & 0.1880 & 0.2 & 1.172 & 1.169 \\
1.1 & 0.3 & 1.5 & 0.8081 & 0.4 & 20.369 & 19.882 \\
-1.6 & 1.2 & 0.7 & -0.4464 & 0.6 & 0.302 & 0.314 \\
-1.3 & -0.5 & -0.7 & -1.7582 & 0.8 & 1.407 & 1.659 \\
\hline
\end{tabular}

\section{GLR Model with Periodically Correlated Errors}

Consider the SLR model (1) with errors $\left\{\epsilon_{t}\right\}$ being periodically correlated following the $\operatorname{PAR}_{\omega}(1)$ model as in (8). Then without loss of generality assuming $n=m \omega$ and denoting the SACVF of $\left\{\epsilon_{k \omega+\nu}\right\}$ by $\gamma_{j}(\nu)$ then the variance-covariance matrix of $\left\{\epsilon_{1}, \ldots, \epsilon_{m \omega}\right\}$ is

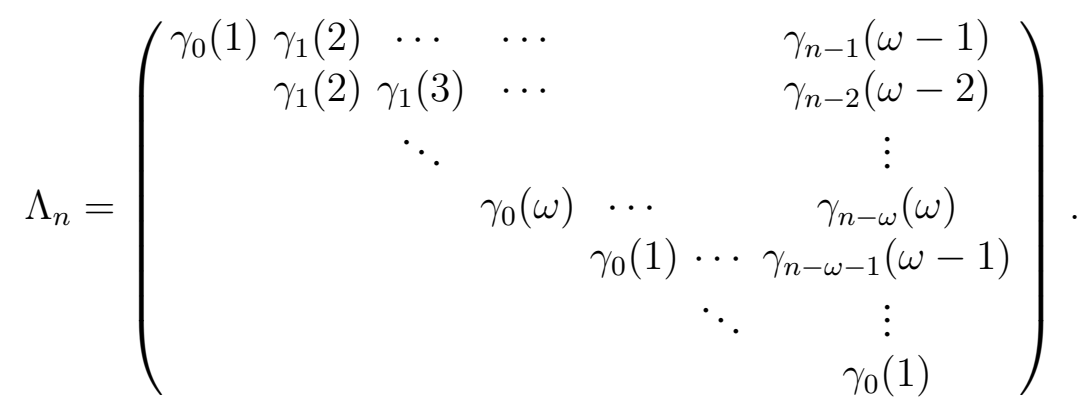

Notice, if $n=m \omega$ the diagonal elements of $\Lambda_{n}$ are $m$ cycles of $\left(\gamma_{0}(1), \ldots, \gamma_{0}(\omega)\right)$. Now, the GLS estimate of $\beta$ as well as its variance are given by (4) and (6), respectively, but $\Lambda_{n}$ replacing $\Gamma_{n}$.

As the theoretical SACVF of $\left\{\epsilon_{t}\right\}$ is unknown, then it is traditionally estimated via the residuals $\left\{e_{t}\right\}$ of the OLS of (1). That is, if $C_{n}$ is the same as $\Lambda_{n}$ above with $C_{j}(\nu)$ 
(defined in (10)) in place of $\gamma_{j}(\nu)$ then

$$
\hat{\beta}_{G L S}=\left(X_{n}^{\prime} C_{n}^{-1} X_{n}\right)^{-1} X_{n}^{\prime} C_{n}^{-1} Y_{n}
$$

and

$$
S^{2}\left(\hat{\beta}_{G L S}\right)=\left(X_{n}^{\prime} C_{n}^{-1} X_{n}\right)^{-1} .
$$

For the $\operatorname{PAR}_{\omega}(1)$ error model, $\gamma_{j}(\nu)$ are computed using Theorem 1 where $\gamma_{0}(\nu)$ is estimated by $C_{0}(\nu)$. For the estimation of $\phi_{1}(\nu)$, in view of (8), it is obtained through regressing (through origin) of the residuals in the $\nu$-th season on the preceding residuals which belong to the $(\nu-1)$-th season.

Example 2: Consider the quarterly time series of US passengers miles (in millions) for Q1, 1996 to Q4, 2004 (Cryer and Chan, 2008) shown in Figure 1 below. This graph shows a nearly linear trend with seasonality. The fitted OLS of the linear trend model is given by $\hat{Y}_{t}=104.608+0.866 t$, so that

$$
\hat{\beta}_{O L S}=\left(\begin{array}{r}
104.608 \\
0.867
\end{array}\right) \quad \text { and } \quad S^{2}\left(\hat{\beta}_{O L S}\right)=\left(\begin{array}{rr}
9.447 & -0.388 \\
-0.388 & 0.021
\end{array}\right) .
$$

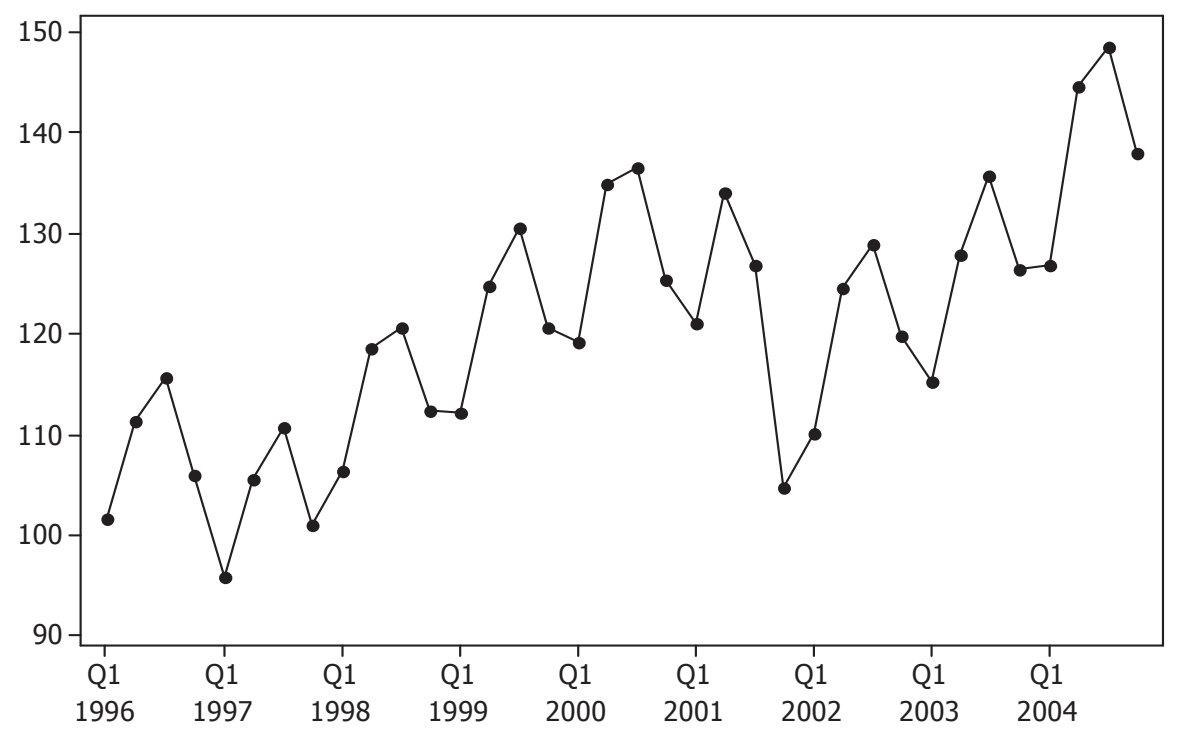

Figure 1: The time series plot of quarterly U.S. airline passenger miles(in millions).

Also the McLeod test for periodic correlations among errors is applied (using Rpackage pear) and is found highly significant ( $p$-value $=0.00009)$. Then based on the residuals of the fitted OLS model, we find that: $\hat{\phi}_{1}(1)=0.868, \hat{\phi}_{1}(2)=-0.043$, $\hat{\phi}_{1}(3)=0.957, \hat{\phi}_{1}(4)=-0.002$, and $C_{0}(1)=33.93, C_{0}(2)=46.27, C_{0}(3)=33.66$, $C_{0}(4)=61.12$. Taking $X_{n}=X_{36}=\left(1_{36}, X_{i}\right)^{\prime}$ with $X_{i}=(1, \ldots, 36)^{\prime}$, computing the matrix $C_{n}$ and applying the formulas above, we get

$$
\hat{\beta}_{G L S}=\left(\begin{array}{r}
105.14 \\
0.875
\end{array}\right) \quad \text { and } \quad S^{2}\left(\hat{\beta}_{G L S}\right)=\left(\begin{array}{rr}
9.246 & -0.386 \\
-0.386 & 0.026
\end{array}\right) .
$$


Finally, we notice that although the estimates and standard errors based on OLS and GLS are close to each others, the OLS results here are invalid and those based on GLS should be more accurate.

In Example 2 above the GLS and OLS estimates are compared for some real data. In fact, the implications of the nature and strength of the autocorrelation structure among errors on the relationship between those estimates is a crucial question. From a design point of view, various studies highlight important issues related to effective design under correlation (see for example Müller and Stehlík, 2009). In the following example we study the effect of the autocorrelation design on the estimates of both methods. Albertson, Aylen, and Lim (2002) studied the power of Durbin-Watson test for SLR models with PAR(1) errors. The selected designs in the next example are due to them.

Example 3: Here we consider the GLR model

$$
Y_{=1}+5 t+\epsilon_{t}, \quad t=1, \ldots, 4 n
$$

where $\left\{\epsilon_{t}\right\}$ is $\operatorname{PAR}_{4}(1)$ defined in (8) with the $\phi$ 's as defined below and $\sigma_{u}^{2}(\nu)=1$, for all $\nu$. We use 1000 realizations of $\epsilon_{t}$ (and thus $Y_{t}$ ) assuming that the $u_{t}$ are iid $\mathrm{N}(0,1)$ each of length 200 (that is 50 years) according to the following cases:

Case 1: $\phi_{1}(1)=\rho / \tau, \phi_{1}(2)=\rho \tau, \phi_{1}(3)=\phi_{1}(4)=\rho$, with $\rho=-0.9,-0.5,0,0.5,0.9$ and $\tau=1,1.1,1.25,1.5,2$.

Case 2: $\phi_{1}(1)=0, \phi_{1}(2)=\rho, \phi_{1}(3)=0, \phi_{1}(4)=\rho$, with $\rho=-2,-1,-0.5,0.5,1,2$.

Table 4: Average relative efficiencies of OLS and GLS estimates for case 1.

\begin{tabular}{rrrrrrr}
\hline$\rho$ & & $\tau=1$ & $\tau=1.1$ & $\tau=1.25$ & $\tau=1.5$ & $\tau=2$ \\
\hline-0.9 & $\beta_{0}$ & 32.245 & 23.878 & 25.698 & 30.967 & 23.188 \\
& $\beta_{1}$ & 32.088 & 23.819 & 25.606 & 30.869 & 23.051 \\
-0.5 & $\beta_{0}$ & 3.293 & 3.302 & 3.357 & 3.586 & 4.187 \\
& $\beta_{1}$ & 3.287 & 3.296 & 3.349 & 3.575 & 4.169 \\
0.0 & $\beta_{0}$ & 1.112 & & & & \\
& $\beta_{1}$ & 1.111 & & & & \\
0.5 & $\beta_{0}$ & 0.388 & 0.390 & 0.394 & 0.404 & 0.462 \\
& $\beta_{1}$ & 0.390 & 0.391 & 0.395 & 0.405 & 0.461 \\
0.9 & $\beta_{0}$ & 0.099 & 0.119 & 0.191 & 0.405 & 1.001 \\
& $\beta_{1}$ & 0.102 & 0.121 & 0.190 & 0.397 & 0.978 \\
\hline
\end{tabular}

Then for each realization the relative efficiency of the OLS and the GLS estimates of $\beta_{0}=1$ and $\beta_{1}=5$ is computed. Then the average relative efficiency based on all realizations is obtained and summarized in Tables 4 and 5. In case 1, the geometric mean of the $\phi$ 's is $\rho$ which represents the degree of correlation while $\tau$ controls the degree of periodicity. Case 2 represents a semi-periodic PAR(1) model. Notice for case 2 that $\rho$ 
Table 5: Average relative efficiencies of OLS and GLS estimates for case 2.

\begin{tabular}{ccccccc}
\hline & $\rho=-2$ & $\rho=-1$ & $\rho=-0.5$ & $\rho=0.5$ & $\rho=1$ & $\rho=2$ \\
\hline$\beta_{0}$ & 14.413 & 4.088 & 2.010 & 0.790 & 0.838 & 3.074 \\
$\beta_{1}$ & 14.368 & 4.082 & 2.009 & 0.789 & 0.832 & 3.030 \\
\hline
\end{tabular}

can assume any real number as the periodic stationarity condition $\left|\prod_{\nu=1}^{4} \phi_{1}(\nu)\right|<1$ is always satisfied.

For case 1, it can be seen that relative efficiency for both intercept and slope are nearly equal. Besides, for negative (positive) autocorrelation, variances due to OLS are larger (smaller) than for GLS. This is true for the semi-PAR(1) model in case 2 . In case 1 , as the degree of periodicity increases the relative efficiency values, generally increase.

\section{Conclusion and Remarks}

In this article we have investigated the properties of the LS estimators of the slope and intercept parameters of the simple linear regression model. We obtained the relative efficiency of those estimators when the errors are correlated via an $\operatorname{AR}(1)$ model as well as when the errors are periodically correlated following the PAR(1) model compared to the standard case of WN errors. An example is provided for various models of those types. The main conclusions are that the LS estimators are still unbiased but the ordinary formulas for $\operatorname{var}\left(\hat{\beta}_{0}\right)$ and $\operatorname{var}\left(\hat{\beta}_{1}\right)$ are not reliable and may underestimate or overestimate the actual variances of those estimators. Those variances are affected by the strength and direction of autocorrelation among errors when the errors follow the $\operatorname{AR}(1)$ and $\operatorname{PAR}(1)$ models and are also affected by the nature of periodic autocorrelation when the errors follow the PAR(1) model. Therefore, the known deficiency of OLS estimates when the errors are $\mathrm{AR}(1)$ also extends to the case of periodically correlated errors. We believe that the conclusions above extend to the multiple regression model. This point can be investigated in a future research.

When the errors are autocorrelated then the ordinary formulas for $\operatorname{var}\left(\hat{\beta}_{0}\right)$ and $\operatorname{var}\left(\hat{\beta}_{1}\right)$ should not be used. In this case, a suitable alternative is using GLS method which is defined here when the errors are periodically correlated. Some other remedial measures can also be applied such as including some seasonal component in the regression model as dummy variables or trigonometric functions.

\section{Acknowledgments}

The authors acknowledge with thanks the comments by an anonymous referee as well as the efforts of the editor that led to an substantial improvement of this paper. 


\section{References}

Albertson, K., Aylen, J., and Lim, K. B. (2002). The Power of the Durbin Watson test when the errors are PAR(1). Journal of Statistical Computation and Simulation, 72, 507-516.

Box, G., Jenkins, G., and Reinsel, G. (1994). Time Series Analysis, Forecasting and Control (3rd ed.). Engelwood Cliffs, NJ: Prentice-Hall.

Choudhury, A. H., Hubata, R., and Louis, R. (1999). Understanding time-series regression estimators. The American Statistician, 53, 342-348.

Cryer, J. D., and Chan, K. (2008). Time Series Analysis with Applications in R (2nd ed.). New York: Springer.

Franses, P., and Paap, R. (2004). Periodic Time Series Models. Oxford: Oxford University Press.

Grenander, U. (1954). On the estimation of regression coefficients in the case of autocorrelated disturbances. Annals of Mathematical Statistics, 25, 252-72.

Huitema, B. E., and McKean, J. W. (2007). An improved portmanteau test for autocorrelated error in interrupted time-series regression model. Behavior Research Methods, 39, 343-349.

Jeske, R., Bütefisch, T., and Song, S. H. (1996). The efficiency of the sample mean in a linear regression model when errors follow a first-order moving average process. Economics Letters, 52, 235-240.

Kutner, H., Nachtsheim, J., Neter, J., and Li, W. (2005). Applied Linear Statistical Models (5th ed.). Chicago:IL: McGraw-Hill/Irwin.

Lee, J., and Lund, R. (2004). Revisiting simple linear regression with autocorrelated errors. Biometrika, 91, 240-245.

McLeod, A. I. (1995). Diagnostic checking periodic autoregression models with application. The Journal of Time Series Analysis, 15, 221-233.

Mohammed, N., and Ibazizen, M. (2008). Bayesian estimation of the regression model with autocorrelated errors under contamination. Communications in Statistics: Theory \& Methods, 37, 2382-2389.

Müller, W. G., and Stehlík, M. (2009). Issues in the optimal design of computer simulation experiments. Applied Stochastic Models in Business and Industry, 250, 163-177.

Obeysekera, J. T. B., and Salas, J. D. (1986). Modeling of aggregated hydrologic time series. Journal of Hydrology, 86, 197-219.

Ohtani, K. (1990). On estimating and testing in a linear regression model with autocorrelated errors. Journal of Econometrics, 44, 333-346.

Rohatgi, V. K. (1984). Statistical Inference. New York: John Wiley and Sons.

Tiao, G., and Grupe, M. (1980). Hidden periodic autoregressive-moving average models in time series data. Biometrika, 67, 365-373.

Ula, T. A., and Smadi, A. A. (1997). Periodic stationarity conditions for periodic autoregressive moving average processes as eigen value problems. Water Resources Research, 33, 1929-1934.

Wei, W. (2006). Time Series Analysis, Univariate and Multivariate Methods (2nd ed.). Boston, MA: Pearson, Addison-Wesley. 
Yue, F., and Koreisha, S. G. (2004). Forecasting with serially correlated regression models. Journal of Statistical Computation \& Simulation, 74, 625-649.

Zinde-Walsh, V., and Galbraith, J. (1991). Estimators of a linear regression model with stationary ARMA(p, q) errors. Journal of Econometrics, 47, 333-357.

Author's addresses:

Abdullah A. Smadi

Department of Statistics

Yarmouk University

Irbid, Jordan

E-Mail: asmadi@yu.edu.jo

Nour H. Abu-Afouna

Department of Mathematics

Princess Noura University

Riyadh, Saudi Arabia

E-Mail: nouraboafouneh@yahoo.com 